\title{
Current Status of and Opinions on Heart Transplantation in China
}

\author{
Yong-feng SUN, Zhi-wen WANG, Jing ZHANG, Jie CAI, Feng SHI", Nian-guo DONG ${ }^{\#}$ \\ Department of Cardiovascular Surgery, Union Hospital, Tongji Medical College, Huazhong University of Science and \\ Technology, Wuhan 430022, China
}

(C) The Author(s) 2021

\begin{abstract}
[Abstract] The purpose of this review is to provide a comprehensive update on recent advances in heart transplantation in China. Despite advances in pharmacologic and device treatment of chronic heart failure, long-term morbidity and mortality remain high, and many patients progress to endstage heart failure. Heart transplantation has become standard treatment for selected patients with end-stage heart failure, though challenges still exist. However, multiple advances over the past few years will improve the survival and quality-of-life of heart transplant recipients. This article elaborates on the specific characteristics of heart transplantation in China, the current issues, development trends, and related experiences with heart transplantation in Wuhan Union Hospital.

Key words: heart transplant; China; current status
\end{abstract}

Heart failure is a cardiovascular disease with an increasing number of cases globally. According to published data, there are approximately 4.5 million patients with heart failure in China ${ }^{[1]}$. End-stage heart failure is that heart failure has reached an irreversible stage. Globally, the number of cases of end-stage heart failure increases by over 800000 cases/ year, and carries with it a 1 -year mortality rate of $70 \%$ and a sudden death rate of $60 \%{ }^{[2]}$. There are no efficacious drug treatments against end-stage heart failure and heart transplantation is currently recognized as the most effective treatment. This article elaborates on the specific characteristics of heart transplantation in China, the current issues, development trends, and related experiences with heart transplantation in Wuhan Union Hospital, China.

\section{CURRENT STATUS AND CHARACTERISTICS OF HEART TRANSPLANTATION IN CHINA}

Heart transplantation began relatively late in China, but has undergone rapid development. With the creation of China's heart transplant registry in 2009 and the establishment of voluntary organ donation system for Chinese citizens in 2015, the number and quality of heart transplants have greatly increased. Within the heart transplant teams at Beijing Fuwai Hospital and Wuhan Union Hospital, the number and treatment efficacy of heart transplantation have gradually become

Yong-feng SUN, E-mail: catch0209@163.com \#Corresponding authors, Nian-guo DONG, E-mail: dongnianguo @hotmail.com; Feng SHI, E-mail: catch0209@163.com aligned with those of more advanced countries. There are currently 57 qualified heart transplantation units in China. As of 2019, a total of 3756 cases of heart transplants were registered (fig. 1).

Due to differences in China's national conditions, economic conditions, and societal values, compared to Europe and the United States (International Society of Heart and Lung Transplantation, ISHLT), Chinese heart transplantation exhibits the following characteristics: 1) Over the past 5 years, doctors in Europe (40\%) and the United States $(60 \%)$ have performed 5500 heart transplants annually ${ }^{[3]}$. In contrast, approximately 500 heart transplants are performed every year in China, less than 1/10 the total number performed in Europe and the United States. As a country with a large population, there is much room for improvement in the number of heart transplants performed. 2) Age distribution, sex, and other basic characteristics of donors and recipients in China are similar to those in

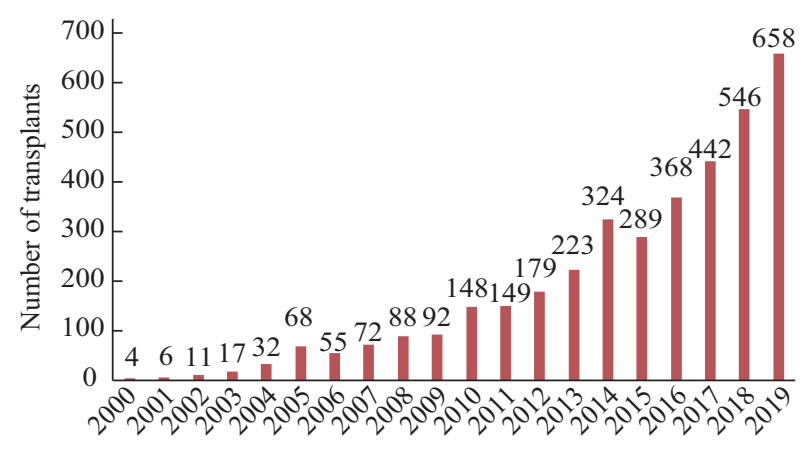

Fig. 1 Number of heart transplants by year in China (2000-2019) 
Europe and the United States. Donors are mainly 1839 years old, while recipients are mainly 40-59 years old. However, in recent years, in western countries, donors over the age of 60 have increased each year, and patient age $>70$ is no longer a contraindication for surgery ${ }^{[4]} .3$ ) Dilated cardiomyopathy is the main cause of disease for the recipient. In Europe and the United States, dilated cardiomyopathy accounts for 50\% of cases, while in China it accounts for $73.9 \%$ of cases, which is relatively high. Ischemic cardiomyopathy accounts for $33 \%$ of cases in Europe and the United States, which is higher than that in China, where it accounts for only $15.1 \%$ of cases. Complex congenital heart disease accounts for $6 \%$ of cases in Europe and the United States among infants $(<1$ year old), which is higher than that in China, where only $3 \%$ of cases fall under this category and the average age of the recipient is 10 years old. Heart transplants after another heart surgery account for $76 \%$ of cases in Europe and the United States, but only $16 \%$ of cases in China (fig. 2). China has a high rate of dilated cardiomyopathy, but a low rate of coronary heart disease and heart transplant after another heart surgery may be important factors for the high survival rate in China. 4) Due to differences in organ transportation systems, $70 \%$ of cases in Europe and the United States receive transplantation with hearts that have undergone cold ischemia time less than $4 \mathrm{~h}$, while the hearts for $52.5 \%$ of cases in China experience cold ischemia time greater than $4 \mathrm{~h}$, and for $28.2 \%$ of cases, the cold ischemia time of hearts is greater than $6 \mathrm{~h}^{[5]}$. Therefore, it is necessary to create a safe organ transportation system. 5) The bridge to transplantation used in Europe and the United States is primarily the ventricular assisted device (VAD), which can generally last for several months to 2 years ${ }^{[6]}$. In China, a new mechanical assisted mode developed for use during the perioperative period uses an intra-aortic balloon pump (IABP) as the main component, and extracorporeal membrane oxygenation (ECMO) as the complementary component. This assisted mode was

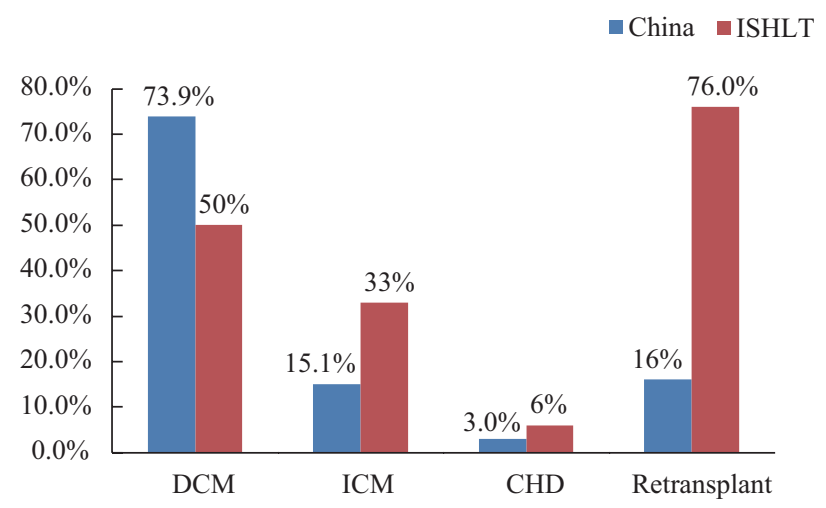

Fig. 2 Heart transplants recipient diagnosis in China DCM, dilated cardiomyopathy; ICM, ischemic cardiomyopathy; CHD, congenital heart disease developed based on China's national conditions and has achieved good clinical results.

\section{MAIN ISSUES WITH HEART TRANSPLAN- TATION IN CHINA}

Although heart transplantation in China has shown great progress, it still faces the following problems: 1) Donor heart shortage. The current organ donation cannot meet the needs of end-stage patients. In 2018, there were 21000 organ donations after death among Chinese citizens for a total of 58000 donated organs. The number of donations and transplants ranked second in the world, and first in Asia, but the donation rate per million people (PMP) is only 4.60, ranking 25 th lowest in the world (The data come from IRODaT Newsletter 2019) (fig. 3). In addition, using China's current classification criteria for organ donation, C-I type donation after brain death (DBD) accounts for only $14 \%$ of total donations, C- II donation after cardiac death (DCD), and C-III donation after brain and cardiac death (DBCD) accounted for $86 \%$, resulting in the loss of a large number of high-quality hearts. After 2015, all heart transplant donors came from DBD. In addition, heart donation mostly depends on liver and kidney organ procurement organizations (OPO). Due to improper donor maintenance, donor/ recipient weight mismatch, infectious diseases, and other factors, a large number of donor hearts cannot be used. 2) Imbalance in the use of critical transplant technologies. There is a significant difference in the number of heart transplantation operations among various hospital units. In 2018, Beijing Fuwai Hospital and Wuhan Union Hospital accounted for half of the national annual number of surgeries, and there were 11 hospitals that performed greater than 10 transplants. The vast majority of hospitals are still only at the initial stage of heart transplantation, with only occasional reports emerging from these units (fig. 4). 3) The organ donation information network distribution system and the green channel developed for the rapid transfer of human organs must be further improved. In 2013, China established the China Organ Transplant Response System (COTRS). In 2016, six government ministries and commissions jointly opened a green channel for human organ donation. The rate of organ sharing nationwide increased by $7.3 \%$, rate of organ utilization increased by $6.7 \%$, and organ transportation time was shortened by $1-1.5 \mathrm{~h}$ (the time for heart transportation was reduced from $4.89 \mathrm{~h}$ to $3.78 \mathrm{~h}$ ). If the distribution process is further regulated to avoid transportation delays, heart utilization can be maximized. 4) Because there is no multicenter statistics, the heart transplantation waiting list (HTx WL) in China is still unknown, but the heart transplantation waiting time according to HTx WL in our center is about 2 months 


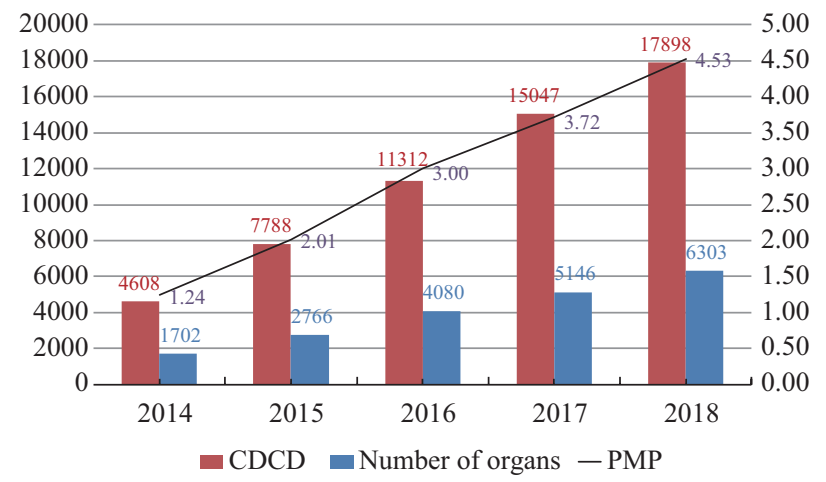

Fig. 3 Number of organ donations in China

CDCD, China donation after citizen's death; PMP, per million population

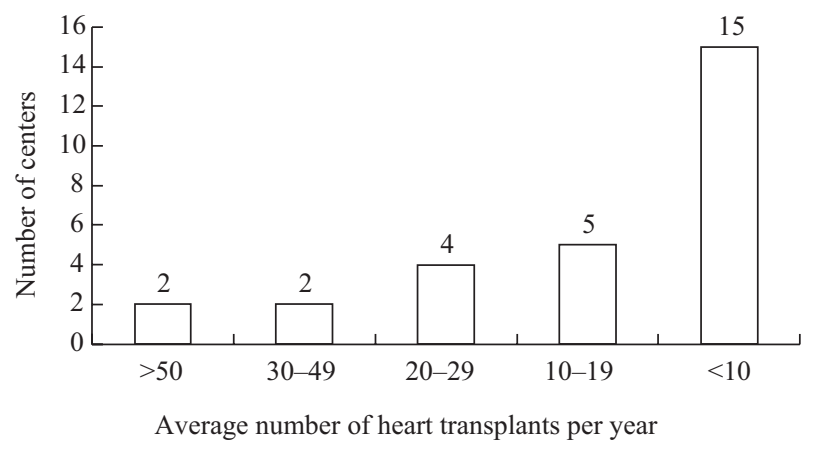

Fig. 4 Heart transplant average center volume

to 3 years. VAD has not been widely used in China, and there is not enough experience in bridging treatment before transplantation. ECMO and IABP are mainly used before heart transplantation. Transplant patients were also affected during the COVID-19 pandemic. According to the data of our centers, this effect was not much different from the normal population. We recommend that transplant recipients should be warned in terms of personal hygiene and should be closely monitored by organ transplant centers. If there is an indication for hospitalization, they should be followed in an isolated unit, with no aggressive changes made to immunosuppressive doses unless necessary. Moreover, novel coronavirus pneumonia does lead to a longer waiting time for organ transplant in 2020. 5) Heart transplantation is not covered by medical insurance in most areas of China. According to published statistics, the cost of heart transplantation in the United States is USD $\$ 990$ 000/case, while in China the cost is RMB $300000 /$ case (nearly $1 / 22$ that of the US cost). The cost of long-term use of immunosuppressive agents is approximately RMB 5000/month. A considerable number of patients abandon treatment because of the excessive financial burden. Incorporating heart transplantation into medical insurance is an important step toward achieving a healthy China 2030 strategy and to improve the level of heart transplantation diagnosis and treatment in China.

\section{FUTURE DEVELOPMENT TREND OF HEART TRANSPLANTATION}

Heart transplantation inherently involves high-risk, multi-disciplinary, and complex surgical techniques. Possible future developments include: 1) widespread use of VAD. With further progress in science and technology and developments in materials science, VAD will certainly become a crucial tool as either a bridge to transplant for end-stage heart failure, an end-point, or a decision-making element during the treatment process. Since 2006, nearly 30000 patients in North America have received a VAD, and greater than 3200 patients received VAD implants in $2013^{[7]}$. The 1-year survival rate is $80 \%$, and the 2 -year rate is $70 \%$, very similar to that of heart transplantation ${ }^{[8]}$. The researcher Shengshou Hu has devoted himself to the research and development of locally manufactured VADs and associated clinical trials adapted to China's unique national conditions. In this area, he has achieved fruitful results. In August 2019, the first artificial heart developed in China, Chongqing Yongrenxin "EVAHEART", was approved for commercial sale. In the same year, the world's smallest magnetic levitation centrifugal artificial heart Suzhou Tongxin " $\mathrm{CH}-$ VAD" entered into clinical trials. More than 30 VADs were implanted at Beijing Fuwai and Wuhan Union Hospitals, with good patient outcomes. 2) Expand the source of donor hearts. With the advancement of myocardial protection technology, donor delivery system, perioperative management, and anti-rejection drugs, marginal donor hearts can be used more and more to treat end-stage heart failure. The clinical application of DCD donor hearts may be an important way to increase the source of donor hearts. The main challenge in using DCD hearts to find a way to reduce warm ischemic injury and ischemia-reperfusion injury. A large number of basic and pre-clinical studies have shown that under $15 \mathrm{~min}$ of myocardial warm ischemia, it is possible to repair damage to the heart. The world's first case of heart transplantation was using a DCD donor heart (warm ischemia time $5 \mathrm{~min})^{[9,10]}$. In recent years, successful cases of DCD donor heart transplantation in children and adults have been reported one after another, providing a basis for evading ethics and achieving DCD donor heart transplantation ${ }^{[10-15]}$. In addition, the upper limit of donor age has been increased, the range of donor/ recipient body weight ratio has been expanded ${ }^{[16,17]}$, and antiviral treatment of hepatitis $\mathrm{C}$ donor hearts can also improve donor heart utilization rate ${ }^{[18,19]} \cdot 3$ ). Noninvasive rejection monitoring. Previously, tissue biopsy was considered to be the gold standard for the diagnosis of rejection, but it has the disadvantage of being an invasive, subjective, and lagging technique, while non-invasive, sensitive, and timely detection methods 
such as coronary CTA (CCTA), immune system gene expression of transplanted patients (AlloMap), cellfree donor-derived DNA (cfdDNA), and noninvasive ultrasound molecular imaging will surely become important methods for monitoring cardiac rejection in the future ${ }^{[20-23]}$.

\section{EXPERIENCE IN HEART TRANSPLANTATION FROM WUHAN UNION HOSPITAL}

With the efforts of several generations of clinicians at Wuhan Union Hospital, great progress has been made in the field of heart transplantation over the past decade. A total of 698 heart transplants have been performed since 2008. Over the past 7 years, the total number of surgeries at Wuhan Union Hospital has been the largest in China, which is ranked among the foremost in the world. The perioperative success rate is $97.6 \%$, and the 1 -year and 5-year survival rates are $90.1 \%$ and $84.0 \%$, which are superior to those reported by the ISHLT. Wuhan Union Hospital also achieved heart transplantation in the country's youngest/smallest infant (66 days $/ 3 \mathrm{~kg}$ ). In 2019, the publication "Development and Application of Key Heart transplantation Technology System" won first prize from the Science and Technology Achievement Award of the Ministry of Education, and in 2010 the publication "Experimental and Clinical Research on Heart and Heart and Lung Transplantation" won first prize from the Hubei Provincial Science and Technology Progress Award. The main highlights from their work are shown as the following.

1) They established a heart transplant recipient evaluation system and a new perioperative mechanical assistance mode. Together with Professor Yunhu Song of Beijing Fuwai, Wuhan Union Hospital led the development of the "Technical Specifications for Pre-Operative Evaluation and Preparation of Chinese Heart Transplant Recipients" by the Chinese Medical Association Organ Transplantation Branch. Doppler ultrasound, MRI, and other means were comprehensively used to establish an automatic calculation method based on curvature/feature tracking (CardiowerkzTM), which provides important myocardial mechanical information for accurate assessment of end-stage heart disease. The mitral valve annulus contraction distance and myocardial curvature index have been used as clinical markers. Combined with Chinese national conditions, through years of clinical exploration, Wuhan Union Hospital established a new perioperative auxiliary mode of "IABP-based, combined application of ECMO" heart transplantation to reduce the postoperative ECMO use rate, reduce cost and improve survival rate. This approach has proven to be very efficacious following widespread national promotion and use.
2) They optimized the DBD heart protection strategy. Wuhan Union Hospital adopted cold crystal induced cardioplegia, histidine-tryptophanketoglutarate solution (HTK) liquid perfusion protection, ice crystal layering preservation, gradient cooling and controlled reperfusion, and other methods to enhance the protection of the donor heart. The number of DBD heart transplants at Wuhan Union Hospital is in the lead in China and the transplant success rate surpasses that of the ISHLT in the near and medium term. Due to the serious imbalance between supply and demand, Wuhan Union Hospital's "marginal heart supply" standard (2011) was developed to expand the age, donor/recipient weight ratio, cold ischemic time, and blood type compatibility for donor-recipient pairs. Researchers have retrospectively analyzed the post-operative survival rate of 371 cases and found no differences between using the marginal heart supply and using the conventional heart supply. This greatly improves the utilization rate of donor hearts and provides a reference for solving the international problem of donor heart shortage. In addition, an innovative myocardial protection strategy developed at Wuhan Union Hospital broke through the safe time limit of donor cold ischemia ( $4 \mathrm{~h}$ extended to $6-8 \mathrm{~h}$ ), resulting in a postoperative survival rate that is not statistically different from that using hearts from conventional donors. F1000Prime included the cold ischemic time study recommendation in "2018Newfinding"[24].

3) Wuhan Union Hospital developed breakthrough technologies in complex heart transplantation and multiple organ transplantation. In order to solve the needs of various special cases of clinical transplantation, the classic transplantation method was improved to target complex anatomies of abnormal pulmonary veins, vena cava reflux and atria, pulmonary artery, aorta, etc. New transplantation methods were designed: novel congenital dextrocardia transplantation (6 cases), one-stop hybrid heart transplantation (3 cases), simultaneous heart transplantation and treatment of extra cardiac abnormality (20 cases), whole heart transplantation ( 2 cases), and other surgical procedures which helped overcome clinical bottlenecks, simplify surgical procedures, and improve treatment outcomes. Wuhan Union Hospital also developed breakthroughs in the technical bottleneck of multiple organ transplantation, carried out combined heart/lung/liver/ kidney transplantation (12 cases), and maintained the record for the long-term survival of combined heart and liver transplantation for greater than 3 years. Over the past ten years, 68 children's heart transplants have been completed, and the number makes Wuhan Union Hospital rank the first in the country (accounting for $1 / 3$ of the total number of transplants in China). These transplants include three cases of patients $<1$ year old (accounting for $42.86 \%$ of infant cases), six cases of 
patients $1-3$ years old (accounting for $60 \%$ of cases in this age range), and 25 cases of $4-10$ years old (52.08\% of cases in this age range) (fig. 5). The perioperative success rate was $100 \%$, and the 1-year and 5-year survival rates were $95.74 \%$ and $93.01 \%$, which is $15 \%$ greater than the value reported by ISHLT. Wuhan Union Hospital also twice broke the record for pediatric heart transplant in the youngest and smallest pediatric heart transplant recipients in Asia (three months $/ 4.3 \mathrm{~kg}, 2014$; 66 days $/ 3.0 \mathrm{~kg}, 2019$ ), and implemented the country's first pediatric twin heart transplant. Based on specific characteristics of pediatric heart transplantation, Wuhan Union Hospital created a technical system suitable for China's national conditions: (1) focusing on pediatric heart supply, relaxing the donor/recipient body weight ratio (0.62-6.25) to expand the source of donor hearts; (2) based on the bicaval anastomosis, trying to remove the pathological left atrium and cut the bilateral pleura to solve the problem of implantation with a larger donor heart; (3) innovating new techniques for congenital dextrocardia and one-stop hybrid heart transplantation to address the complex anatomy of congenital heart disease; (4) other strategies including optimizing ECMO management, delaying chest closure in stages, and infant kidney replacement therapy to promote the development of Chinese pediatric Heart transplantation.

4) They discovered new targets and mechanisms associated with transplantation immune tolerance and chronic transplant vascular pathologies. (1) IRF4 was found to inhibit PD-1 expression through an epigenetic mechanism, blocking PD-1 in vivo to reverse T cell incapacitation and induce heart transplant tolerance ${ }^{[25]}$. (2) The mechanism in the regulation of CAV by the HMGB1/TGF- $\beta$ loop was elucidated, confirming the protective effect of TGFBR1 inhibitors in intimal hyperplasia of arteries and the role of smooth muscle precursor cells in maintaining the stability of blood vessel walls, and a model of early injury-induced smooth muscle cell migration was proposed, which promotes artery narrowing. (3) Removal of donorderived MyD88-CXCL2 signaling pathway-dependent non-classical monocytes can alleviate graft dysfunction

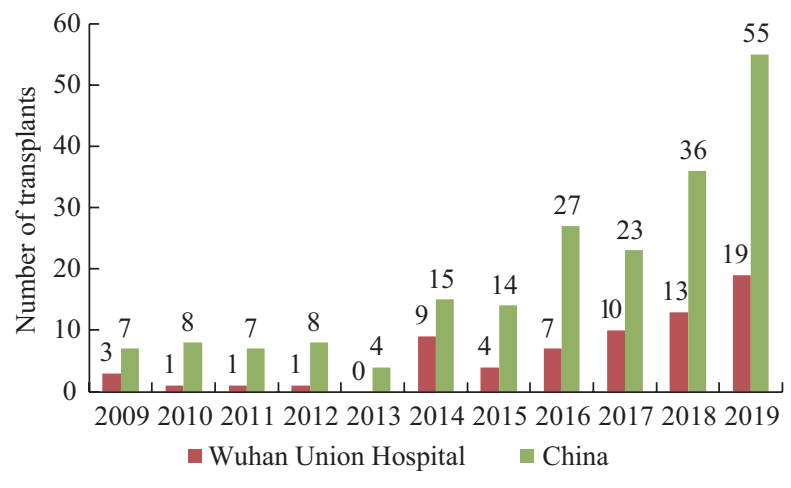

Fig. 5 Number of pediatric heart transplants by year in Wuhan Union Hospital, China and provide new pathways to improve the protection of donor hearts and lungs. (4) CD25 monoclonal antibody induces immune tolerance in heart transplantation. In 2003, CD25 monoclonal antibodies were first used in the clinic. Subsequently, the incidence of acute rejection decreased from $16.7 \%$ in $2003-2008$ to $4.0 \%$ after 2010 .

\section{CONCLUSIONS}

Heart transplantation is continuing to evolve with exciting new advancements in the preoperative, perioperative, and postoperative management of heart transplantation patients. For carefully selected patients, heart transplantation markedly improved survival and quality-of-life. In the years to come, limitations in donor organ availability and preservation, along with immunosuppression, will be important areas for improvement. The advances outlined here, from efforts to expand the donor pool, revision of the heart transplantation allocation policy, and newer ways to diagnose rejection, developments in the detection and care of sensitized heart transplantation candidates, will bring about improvements in survival and quality-oflife of end-stage patients who have heart transplantation.

\section{Open Access}

This article is licensed under a Creative Commons Attribution 4.0 International License https://creativecommons.org/licenses/by/4.0/), which permits use, sharing, adaptation, distribution and reproduction in any medium or format, as long as you give appropriate credit to the original author(s) and the source, provide a link to the Creative Commons licence, and indicate if changes were made. The images or other third party material in this article are included in the article's Creative Commons licence, unless indicated otherwise in a credit line to the material. If material is not included in the article's Creative Commons licence and your intended use is not permitted by statutory regulation or exceeds the permitted use, you will need to obtain permission directly from the copyright holder. To view a copy of this licence, visit http://creativecommons. org/licenses/by/4.0/.

\section{Conflict of Interest Statement}

The authors have declared that they have no conflicts of interest.

Author Nian-guo DONG is a member of the Editorial Board for [Current Medical Science]. The paper was handled by the other Editor and has undergone rigrous peer review process. Author Nian-guo DONG was not involved in the journal's review of, or decisions related to, this manuscript.

\section{REFERENCES}

1 Hu SS, Gao RL, Liu LS, et al. Summary of the 2018 Report on Cardiovascular Diseases in China. Chin Cir J (Chinese), 2019,34:209-220

2 van der Meer P, Gaggin HK, Dec GW. ACC/AHA Versus ESC Guidelines on Heart Failure: JACC Guideline 
Comparison. J Am Coll Cardiol, 2019,73(21):27562768

3 Hayes DJ, Cherikh WS, Chambers DC, et al. The International Thoracic Organ Transplant Registry of the International Society for Heart and Lung Transplantation: Twenty-second pediatric lung and heart-lung transplantation report-2019; Focus theme: Donor and recipient size match. J Heart Lung Transplant, 2019,38(10):1015-1027

4 Mehra MR, Canter CE, Hannan MM, et al. The 2016 International Society for Heart Lung Transplantation listing criteria for heart transplantation: A 10-year update. J Heart Lung Transplant, 2016,35(1):1-23

5 Wang Y, Cai J, Sun Y, et al. Extended donor criteria in heart transplantation: a retrospective study from a single Chinese institution. J Thorac Dis, 2018,10(4):21532165

6 Guglin M, Zucker MJ, Borlaug BA, et al. Evaluation for Heart Transplantation and LVAD Implantation: JACC Council Perspectives. J Am Coll Cardiol, 2020,75(12):1471-1487

7 Kormos RL, Cowger J, Pagani FD, et al. The Society of Thoracic Surgeons Intermacs database annual report: Evolving indications, outcomes, and scientific partnerships. J Heart Lung Transplant, 2019,38(2):114126

8 Rose EA, Gelijns AC, Moskowitz AJ, et al. Long-term use of a left ventricular assist device for end-stage heart failure. N Engl J Med, 2001,345(20):1435-1443

9 Massad M G. Christiaan Neethling Barnard (19222001). J Thorac Cardiovasc Surg, 2002,123(1):1-2

10 Iyer A, Gao L, Doyle A, et al. Normothermic ex vivo perfusion provides superior organ preservation and enables viability assessment of hearts from DCD donors. Am J Transplant, 2015,15(2):371-380

11 Dhital KK, Iyer A, Connellan M, et al. Adult heart transplantation with distant procurement and ex-vivo preservation of donor hearts after circulatory death: a case series. Lancet, 2015,385(9987):2585-2591

12 Boucek MM, Mashburn C, Dunn SM, et al. Pediatric heart transplantation after declaration of cardiocirculatory death. N Engl J Med, 2008,359(7):709-714

13 Messer S, Page A, Axell R, et al. Outcome after heart transplantation from donation after circulatorydetermined death donors. J Heart Lung Transplant, 2017,36(12):1311-1318

14 Kleinmahon JA, Patel SS, Auerbach SR, et al. Hearts transplanted after circulatory death in children: Analysis of the International Society for Heart and Lung Transplantation registry. Pediatr Transplant, 2017,21(8)

15 Chew HC, Iyer A, Connellan M, et al. Outcomes of Donation After Circulatory Death Heart Transplantation in Australia. J Am Coll Cardiol, 2019,73(12):1447-1459

16 Kransdorf EP, Kittleson MM, Benck LR, et al. Predicted heart mass is the optimal metric for size match in heart transplantation. J Heart Lung Transplant, 2019,38(2):156-165

17 Holzhauser L, Imamura T, Bassi N, et al. Increasing heart transplant donor pool by liberalization of size matching. J Heart Lung Transplant, 2019,38(11):11971205

18 Woolley AE, Singh SK, Goldberg HJ, et al. Heart and Lung Transplants from HCV-Infected Donors to Uninfected Recipients. N Engl J Med, 2019,380(17): 1606-1617

19 Radzi Y, Shezad M F, Danziger-Isakov L, et al. Using hepatitis $\mathrm{C}$ and $\mathrm{B}$ virus-infected donor organs for pediatric heart transplantation. J Thorac Cardiovasc Surg, 2019,158(2):548-553

20 Costanzo MR, Dipchand A, Starling R, et al. The International Society of Heart and Lung Transplantation Guidelines for the care of heart transplant recipients. J Heart Lung Transplant, 2010,29(8):914-956

21 Yamani MH, Taylor DO, Rodriguez ER, et al. Transplant vasculopathy is associated with increased AlloMap gene expression score. J Heart Lung Transplant, 2007,26(4):403-406

22 De Vlaminck I, Valantine HA, Snyder TM, et al. Circulating cell-free DNA enables noninvasive diagnosis of heart transplant rejection. Sci Transl Med, 2014,6(241):241ra77

23 Wever-Pinzon O, Romero J, Kelesidis I, et al. Coronary computed tomography angiography for the detection of cardiac allograft vasculopathy: a meta-analysis of prospective trials. J Am Coll Cardiol, 2014,63(19):19922004

24 Wang Y, Cai J, Sun Y, et al. Extended donor criteria in heart transplantation: a retrospective study from a single Chinese institution. J Thorac Dis, 2018,10(4):21532165

25 Wu J, Zhang H, Shi X, et al. Ablation of Transcription Factor IRF4 Promotes Transplant Acceptance by Driving Allogenic CD4+ T Cell Dysfunction. Immunity, 2017,47(6):1114-1128

(Received Sep. 4, 2020; accepted Dec. 1, 2020) 